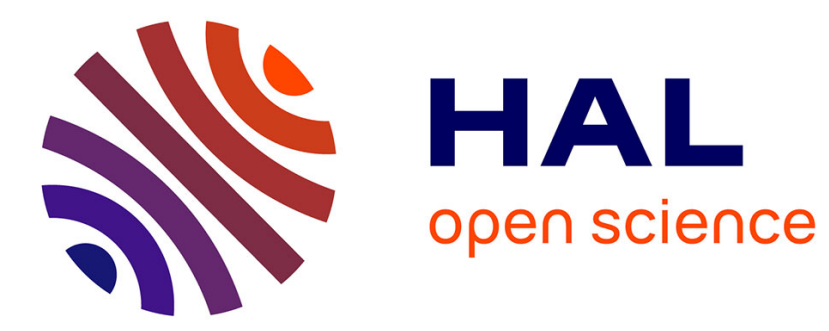

\title{
Dynamics of Spatio-Temporal Binding in Rats
}

\author{
Aurore Malet-Karas, Marion Noulhiane, Valérie Doyère
}

\section{To cite this version:}

Aurore Malet-Karas, Marion Noulhiane, Valérie Doyère. Dynamics of Spatio-Temporal Binding in

Rats. Timing \& Time Perception, 2019, 7 (1), pp.27-47. 10.1163/22134468-20181124 . hal-02194906

\section{HAL Id: hal-02194906 \\ https://hal.science/hal-02194906}

Submitted on 8 Dec 2020

HAL is a multi-disciplinary open access archive for the deposit and dissemination of scientific research documents, whether they are published or not. The documents may come from teaching and research institutions in France or abroad, or from public or private research centers.
L'archive ouverte pluridisciplinaire HAL, est destinée au dépôt et à la diffusion de documents scientifiques de niveau recherche, publiés ou non, émanant des établissements d'enseignement et de recherche français ou étrangers, des laboratoires publics ou privés. 
5 IUniversité Paris-Saclay, Univ Paris-Sud, CNRS, UMR9197, Institut des Neurosciences Paris-Saclay, F 91405, Orsay, 6 France; (2) CNRS, Orsay, F-91405 UMR 9197, Orsay, France; ${ }^{2}$ CNRS, Orsay, France; ${ }^{3}$ 7 UMR 663 INSERM Paris Descartes CEA NeuroSpin/UNIACT, Paris, France

9 *Corresponding author: Valérie Doyère, $\mathrm{PhD}$

10 Université Paris-Saclay, Univ Paris-Sud, CNRS, UMR9197, Institut des Neurosciences Paris-Saclay, France 1191405 , Orsay, France; e-mail : valerie.doyere@u-psud.fr

13 Number of pages: 27

14 Number of figures: 6

15 Number of words: abstract (166); main text (7975)

Keywords: spatio-temporal binding, interval timing, nose poke, peak interval procedure

18 The authors declare no competing financial interests.

Acknowledgements: This work was supported by grants from Agence Nationale de la Recherche (ANR). 
24 Time and space are commonly approached as two distinct dimensions, and rarely combined together in a single task, preventing to compare their interaction. In this project, using a version of a timing task with a spatial component, we investigate the learning of a spatio-temporal rule in animals. To do so, rats were placed in front of a 5-hole nose-poke wall in a Peak Interval (PI) procedure to obtain a reward, with two spatio-temporal combination rules associated with different 29 to-be-timed cues and lighting contexts. We report that, after successful learning of the discriminative task, a single Pavlovian session was sufficient for the animals to learn a new spatiotemporal association. This was evidenced as a beneficial transfer to the new spatio-temporal rule, as compared to control animals that did not experience the new spatio-temporal association during the Pavlovian session. The benefit was observed until 9 days after. The results are discussed within the processes.

\section{Introduction}

Can we access time without having another dimension associated to it, such as an action, or space, or a sequential event? Aristotle proposed that the spatial and temporal contingencies are 40 intrinsically linked together, and cannot be dissociable. Since the $19^{\text {th }}$ century, with the emergence of behavioral experiments, several scientists studied the interaction of these two dimensions; for example, Piaget found that children base their judgements of time and duration on their experience of distance (Piaget, 1927/1969). Fairly recent data confirm that representation of duration is expressed through distance notions, but not the reverse, showing that space and time are asymmetrically dependent on each other (Casasanto and Boroditsky, 2008). To what extent these are bound together, in terms of learning and adaptation to spatio-temporal changes is not yet clear. 
Skinner pointed out the difficulty to experimentally dissociate between where and when an

event occurred, as the memory of the event itself is built on this very association (Skinner 1956; Meck et al, 1984; Crystal, 2009; Meck et al, 2013; Church, 2013 for a more recent review see Church 2014). Many studies have focused on the animal's abilities to learn, consolidate and retrieve spatial or temporal information, although to a lesser extent for the latter, disregarding the potential involvement of the other (space or time) parameter. Spatial memory in animals has been extensively studied through the use of arena exploration, in radial maze (Olton et al, 1977) and the Morris water maze (Morris et al, 1986) in rodents, and many other more complex and ethological experimental setups, such as arena with food cached for monkeys (Lavanex and Lavanex, 2006), rodents (Jacobs and Limans, 1991; Jacobs 1992; Davenport et al, 2000; Gould et al 2010), and even pigs (Mendl et $\mathrm{Al}, 1997)$. In comparison, learning of the temporal dimension has been less investigated. Mainly, time processing has been assessed either through the sequential order of events (Fortin et al, 2002; Kesner et al, 2002), or through the time interval (the duration of a segment of time) (Church et al, 1976; Holders and Roberts, 1985; for a review see Buhusi and Meck, 2005). In a Pavlovian task combining reinforcement time with locations and reinforcement probabilities, a recent study indicates that mice could learn to associate time intervals and locations in order to optimize their behavior (Tosun et al, 2016). However, it is not known to what extent this behavior is flexible when new spatio-temporal rules are learned, and to what extent time and location are bound in memory. dissociated. Indeed, the ability to learn the spatio-temporal variability of important events can confer considerable advantages to animals in the context of both resources seeking as well as predators' avoidance (Crystal 2009). The associative learning of space and time is also referred to as place-time learning, and it has been demonstrated possible with classical conditioning in animals (Wilkie et al, 1997; Thorpe and Wilkie, 2002). Gallistel (1990) proposed that whenever a biologically significant event occurs, a memory code is created that contains the nature of the event, 
as well as the time and place in which it occurred, constituting a tripartite memory. However, still, very little is known regarding the learning of a spatio-temporal (-event or -reward) memory.

Animals can learn time fast (Davis et al, 1989; Bevins and Ayres, 1995; Balsam et al, 2002; Diaz-Mataix et al, 2013), and adapt rapidly (i.e. within a few sessions) to changes in temporal rules in a peak interval protocol (Lejeune et al, 1997; Guilhardi et al, 2005; Sanabria et al, 2014; Dallérac et al, 2017). Similarly, animals can also adapt quickly to changes in a spatial rule from session to session (Morris et al, 1986; Blanco et al, 2006). Tosun et al. (2016) demonstrated that animals can make Bayesian inferences based on spatio-temporal knowledge they previously learned to optimize their time-switching behavior to get the food reward. How the animal learns these two dimensions, and to what extent they are independent of each other, remains unclear.

The aim of the present study was to investigate the dynamics of learning location and time when associated together. For this purpose, learning of spatio-temporal rules was manipulated in a memory paradigm including 3 phases (See Figure 1 for the experimental design): (phase 1) Instrumental training phase of specific spatio-temporal rules associated to different contexts: a Peak Interval (PI) task combining the learning of contextual (different house lights and tone frequencies), spatial (nose poke) and temporal (peak interval) information; (phase 2) Spatiotemporal shift in a single Pavlovian session to control the amount of exposure to stimuli and reinforcement during the learning of a new spatio-temporal rule: some rats were shifted to a new spatio-temporal association (new location and new time) specific to one context using a Pavlovian paradigm, while the others were kept to the trained spatio-temporal rule; (phase 3)

Instrumental training following the new spatio-temporal rule to assess the impact of the preceding session and the dynamic of learning a spatio-temporal association: during nine days, the new spatio-temporal rule was enforced for all animals under the PI procedure. We expected that the rat exposed to the shift in phase 2 will benefit from this training, and show a better learning of the new spatio-temporal rule in phase 3 than the control group. Our analysis aimed at determining 
97 whether the benefit is observed for either location or time, or both, and at deciphering to what

98 extent the learning of new location and new time are linked.

100 Material and Method

101 Animals

10212 adult male Sprague-Dawley rats (Janvier, France; average weight 320g) were housed in pairs in 103 Plexiglas cages, with food and water ad libitum. After one week of adaptation, food access was restricted to 104 keep the animals at $85 \%$ of their normal weight. The experiment was performed in accordance with the recommendations of the European Economic Community (86/609/EEC) and the French National Committee (87/848) for care and use of laboratory animals.

refaite

\section{Figure 1: Experimental design}

(A) Phase 1: Training / Experimental paradigm for the learning of the spatio-temporal association: in context A (red light), the first nose-poke in the hole\#2 lit (empty circle) after $15 \mathrm{~s}$ after the onset of a $1 \mathrm{kHz}$ tone is rewarded (1 pellet), with a maximum tone duration of $45 \mathrm{~s}$ (also duration of probe non-reinforcement trialsreinforcement trials), whereas in context B (green light) the spatio-temporal rule is hole\#4 reinforced after 60 s and a maximum tone duration of 120s. (B) Phase 2: Pavlovian spatio-temporal shift/ The experimental group is presented a Pavlovian session with a new spatio-temporal rule in context B (Hole\#1 lit is reinforced with 3 pellets at 30s after tone onset), while the control group is presented the previous rule (hole\#4, reinforced at 60s). Both groups are then presented the intact context A (hole\#2 lit, reinforced at 15s) in a Pavlovian manner as well. (C) Phase 3: Dynamic of spatio-temporal learning (days 1 to 9 after phase 2). Both groups are presented the new rule (hole\#1, 30s) in context B, while the paradigm associated to context A (hole\#2, 15s) remains the same as in initial training. For these sessions, all the nose-poke holes are lit.

\section{Apparatus}

The experiment took place inside cages (Rat Test Cage, Coulbourn Instruments, USA; dimensions in $\mathrm{cm}$ : 30Lx25lx30h) with a 5-holes nose-poke wall on the left (each hole being $2.5 \mathrm{~cm}$ of diameter and spaced by $2 \mathrm{~cm}$, « 5-holes nose poke », Coulbourn Instruments), a magazine $(4 \times 3 \times 3 \mathrm{~cm})$ connected to a feeder on the opposite side to deliver the reward (45 mg pellets, Rodent Grain-Based Diet, Bioserv). The magazine was lit when the reward was delivered. Two house-lights were the only light source in the cage during the trials, one 
green and one red placed at two distinct extremities of the cage, and served as contextual cues in addition to auditory cues- see below. A speaker was placed on the top of the chamber for delivery of sounds as timing cues. Each of the 5 holes and the magazine were equipped with photoelectric cells to register the activity (nose-poke, NP) of the animals. The cages were placed into a ventilated (background noise, 65dB) soundproof box. The experiment was monitored by the software Graphic State (Coulbourn instruments, USA).

\section{Protocol}

\section{Phase 1: Training}

Pre-training: On the first day of the experiment, the rat was placed into the operant box for a $30 \mathrm{~min}$ session on Continuous Reinforcement (CRF). At the beginning of the session, two pellets were placed into each of the 5 lit holes of the nose-poke wall to encourage the animal to visit them. Each time the animal entered one of the holes, the magazine lit and a pellet was delivered. When the animal entered its nose in the magazine, the light switched off and a new trial began.

Training: The aim of this phase was to train the animals to learn a complex spatio-temporocontextual rule. From day 2 for 41 sessions ( 8 weeks), the animals were submitted to a Peak-Interval (PI) procedure in which they were presented with two different spatio-temporal rules, each associated with a specific context, presented each day in a pseudo randomized order (A followed by B, or B followed by A). Those two contexts were made distinguishable by a house light, red for context A vs. green for context B, and two different timing cues $(1 \mathrm{kHz}-80 \mathrm{~dB}$ and $11 \mathrm{kHz}-80 \mathrm{~dB}$ tones, for $\mathrm{A}$ and B respectively). Each context was associated with a spatial rule indicated by a light in one of the nose-poke holes (hole\#2 in A and \#4 in B). The reward was delivered according to a different Fixed-Interval (FI) in each context (15s after tone onset in $\mathrm{A}$, and $60 \mathrm{~s}$ after tone onset in $\mathrm{B})$ : the first nose-poke after the time into the appropriate hole triggered the delivery of reinforcement and switched on the magazine light and turned off the tone. Probe trials, in which no reinforcement delivery was programmed, consisted of $45 \mathrm{~s}$ for the tone associated with the 
149 FI 15s, and 120s for the FI 60s. Thus, the animal learnt two spatio-temporal rules, each associated to a specific context (context A: hole\#2-15s; context B: hole\#4-60s, see figure 1A).

In order to keep the animal motivated at the beginning of training, we began with 8 Probe trials for 49 reinforcement trials in each context (the first trial in each context was always reinforced), for 10 sessions. 153 Then, we used a ratio of 24 Probe for 37 reinforcement trials until the 8 weeks of training were completed. 154 The inter-trial-interval (ITI) randomly varied between 5 and 30 seconds ( $15 \mathrm{~s}$ on average) in the context A 155 and between 10 and 30 seconds (20 s on average) in B.

\section{Phase 2: Shift of the spatio-temporal association}

In this part of the experiment (day 41), two groups ( $\mathrm{n}=6$ ) were formed, and equilibrated according to their performances. All rats were submitted to a single session during which 3 pellets were delivered in a Pavlovian manner at the end of the timing cue, independently of the animal's behavior. We began with 8 trials in context $\mathrm{B}$ (figure $1 \mathrm{~B}$, right side), during which the control group received the rewards according to the previously learnt association (hole\#4 lit - 60s), whereas the experimental group was submitted to a shift of this associative rule, with the rewards delivered in another hole at another time (hole\#1 lit - 30s). Immediately following this phase, 8 trials rewarded with 3 pellets in context A were also presented in a Pavlovian manner, while keeping its corresponding spatio-temporal rule (hole\#2 lit-15s, figure 1B, left side).

\section{Phase 3: Dynamic of spatio-temporal learning}

The Phase 3 was designed to assess, in the 9 following days, the impact of the single Pavlovian session during which the spatio-temporal rule was shifted, through the measurement of the behavioral adaptation to this new rule in the instrumental task. Therefore, in the context B, only the new rule hole\#1 -

17130 s was reinforced (all holes were lit) while in the context A the rules remained unchanged (hole\#2, 15s). A

172 rapid learning of the spatio-temporal We hypothesized thatthe experimental group would benefit from the previous exposure to the new spatio-temporal rule, and will show better or faster adaptation in comparison to 
174 the control group. The duration of the Probe trials remained as in phase 1 . The first day after phase 2, the rats

175 were submitted to context B first, and then to context A, but the order was pseudo-randomized for the 176 following sessions.

179 Statistical analyses

180 Only the unreinforced probe trials were used to analyze the behavior of the animals. The spatial 181 behavior was assessed by measuring for each rat the percentage of number of nose-pokes (NP) in each hole, 182 then averaged per group. The temporal behavior associated to each hole was analyzed by plotting the 183 function for rate of response (NP) against 1s time bins in context A and 2 s time bins in context B. For comparison of temporal behavior between holes for the sessions after the shift, the temporal activity was normalized by the overall activity of the rats for the hole of interest.

The statistical analyses were performed by SPSS, JASP and Prism. Frequentist ANOVAs Student ttests were used to assess the spatial and temporal learning. We used an alpha level of .05 for all statistical tests. Bayesian ANOVAs were further conducted to better characterize findings in the temporal behavior.

\section{Results}

\section{Training phase}

The temporal behavior associated to each hole in each context over the course of the 8 weeks of training of phase 1 is shown in figure $2 \mathrm{~A}$ and B. A differential behavior associated to each spatio-temporal rule developed progressively with training up to a well segregated temporal behavior, with numbers of nose-poke peaking around 15s in Hole \#2 in context A and around 60s in

Hole \#4 in context B. The spatial behavior was also well segregated, as shown in figure $2 \mathrm{C}$ and $\mathrm{D}$. 
197 In effect, during the last week of training (week 8), the number of visits for the holes of interest of each context were significantly higher than for the other holes, as revealed by the Paired t-tests analyses: the Hole \#2 was more visited in context $\mathrm{A}(\mathrm{NP}=88 \%, \mathrm{SE}=2.7)$ than in $\mathrm{B}(\mathrm{NP}=3.8 \%$,

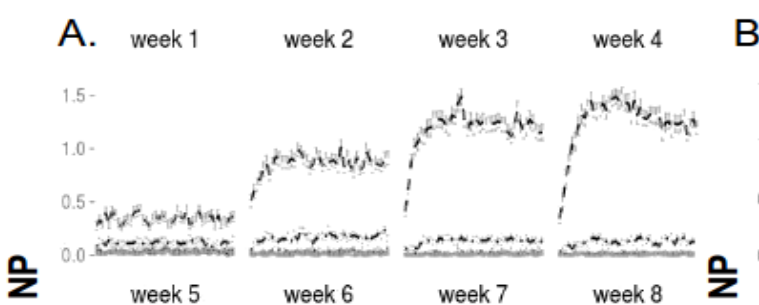

B. week 1 week 2 week 3 week 4

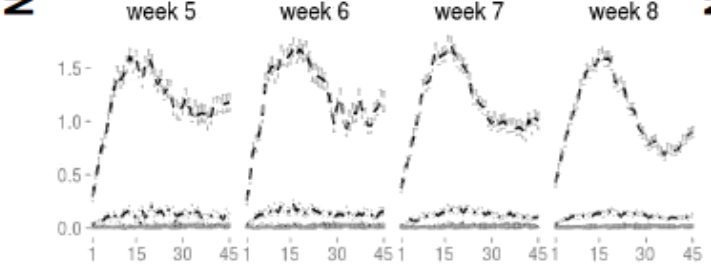

Time (s)
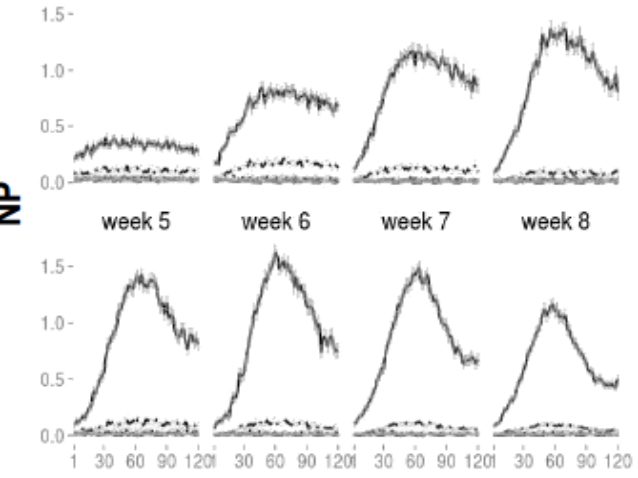

Context A

Time (s)
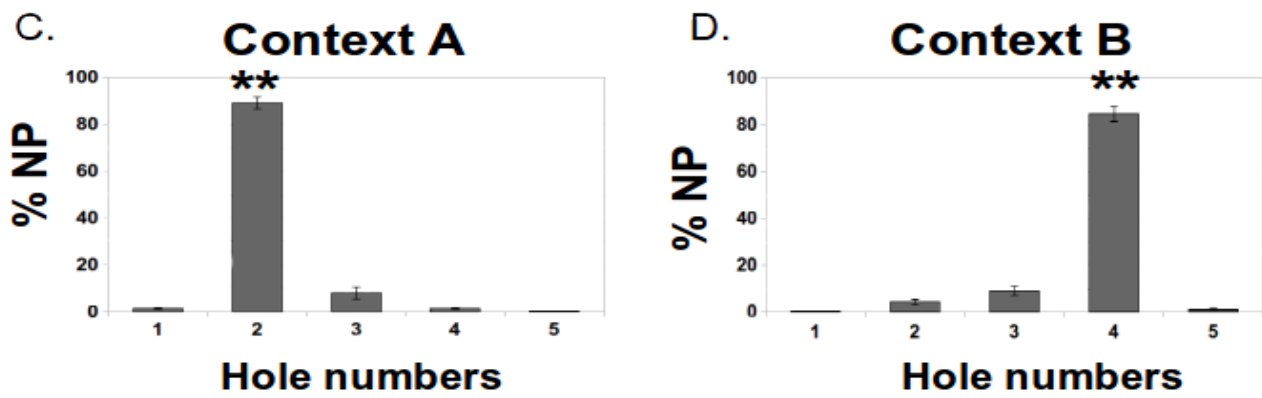

Hole numbers

Figure 2: spatio-temporal training context B with nose-poke reinforced if in hole\#4 after 60s after tone onset (B). Number of Nose-Poke (NP) (+/-SE in grey ribbon) in function of time in context $\mathrm{A}(\mathrm{C})$ and in context B (D). ** $p<.001$ compared to all the other holes. 

temporal rule in context B only, from 60s-Hole\#4 to 30s-Hole\#1, in a Pavlovian session. The other half (control group) were also submitted to a Pavlovian session, but keeping the initial spatiotemporal association. From day 1 to day 9 of Phase 3, the new spatio-temporal (30s-Hole\#1) rule expected to be observable during the next session (expecting a benefit for the experimental group), when both groups were returned to the peak interval procedure with the new spatio-temporal rule enforced in context B and all the holes lit. The analysis of behavior on day 9 aimed to determine whether the benefit might have weakened because both groups were further trained under the new FI schedule.

From day 1 to day 9 of Phase 3, the new spatio-temporal (30s-Hole\#1) rule was enforced in context B for both groups. The impact of Phase 2 (Shift of the association) was assessed during the first session of the instrumental task (day+1), expecting a benefit for the experimental group. As shown in figure $3 \mathrm{~A}$, the spatial behavior of the animals at Day+1 was distributed over several holes, and clearly differed from their behavior at the end of training during Phase 1 (see figure $2 \mathrm{D}$ ). We focused our analyses on three of the holes, the new and old reinforced holes (\#1, \#4, respectively), as well as the hole reinforced in the other context (\#2). There was no difference between groups for any of these holes $(t(10)<1.12$, ns). Compared to their behavior in Phase 1 , the animals decreased significantly their visits in the old hole \#4 $(t(10)=-17.84, p<.001)$, and increased their visits in the new hole \#1 $(t(10)=9.56, p<.001)$. However, even if the hole \#2 was not reinforced in this context, we also observed a significant increase of visits of this hole $(t(10)=8.74, p<.001)$. Surprisingly, the 234 hole \#3, which had never been reinforced, was more visited by the animals now than during the 
235 phase $1(t(10)=4.11, p<.05)$. In contrast, the number of visits in the hole \#5 remained low, and not 236 significantly different from phase $1(t(10)=1.65, \mathrm{~ns})$.

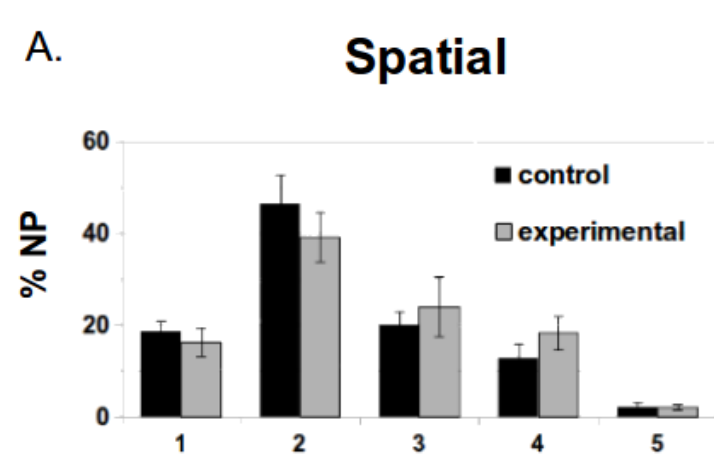

Hole numbers

C.

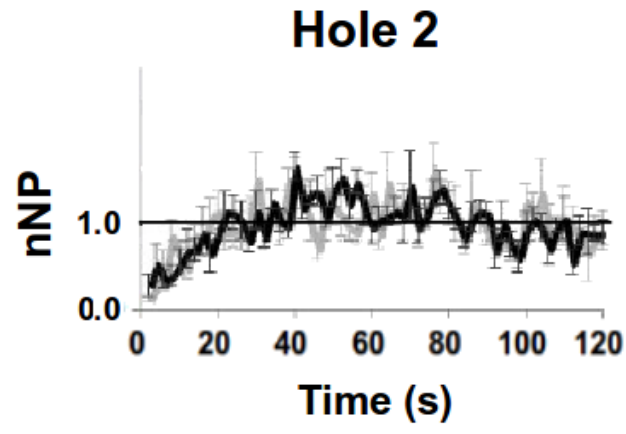

B.

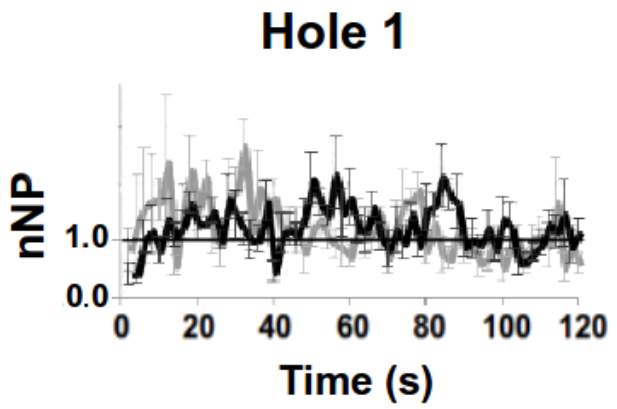

D.

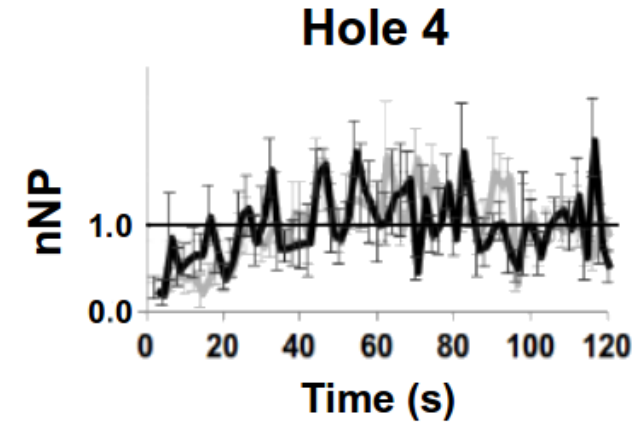

Figure 3: Impact of the Shift session at Day+1 in context B

(A) Spatial activity (\%NP +/- SE) at day 1 after the shift session for all the holes in context B. Temporal behavior for the two groups in the newly reinforced hole \#1, now reinforced at 30s (B), the reinforced hole in the other context, hole \#2 (C) and the previously reinforced hole \#4 (D). The temporal behavior is normalized by the mean activity in the corresponding hole. Control group in black and experimental group in grey. hole\#2, \#3 and \#4, respectively), with no group $\mathrm{x}$ time interaction $(F(59,590)<1.02$ for all, ns). Interestingly, the animals tended to visit these holes with a maximum activity centered at the old 60-s temporal rule. The temporal pattern in hole \#3 was similar to hole \#2 and \#4 (data not shown), 
250

confirming the spatial generalization. The lack of differential behavior between groups was confirmed with Bayesian analyses: a JZS Bayes factor ANOVA with default prior scales revealed that the main effect model was preferred to the group $\mathrm{x}$ time interaction model by a Bayes factor of 30.82 for hole \#2, 6.36 for the hole \#3, and 22.74 for the hole \#4.

Importantly however, the two groups showed a differential temporal behavior in the hole \#1 (significant group $\mathrm{x}$ time interaction, $F(59,590)=1.44, p=.021)$, with the activity of experimental animals peaking earlier, according to the new 30-s temporal rule (figure 3B). Additionally, a more refined analysis of this Day+1 session revealed that only the animals exposed to the new rule in phase 2 presented an adjusted spatio-temporal binding to this new rule. As shown in figure 4A, the temporal behavior of the control animals did not differ significantly in the new (\#1) and old (\#4) reinforced holes (no hole $\mathrm{x}$ time interaction: $F(59,590)=1.14, p=.22$ ), suggesting that the animals did not adapt to the change of rule. Bayesian Repeated measure ANOVA confirmed that the main effect model was preferred to the hole $\mathrm{x}$ time interaction model by a Bayes factor of 7.81 . In contrast, the behavior of experimental animals did differ temporally between those two holes (interaction hole $\mathrm{x}$ time: $F(59,590)=2.16, p<.001$ ) (figure 4B). Bayesian statistics further confirmed this result, as the hole $\mathrm{x}$ time interaction model was preferred to the main effects by a Bayes factor of 9811.33. The animals visited the new hole (\#1) at the new time (30s) and additionally kept the old spatio-temporal association, visiting the old hole more around the old time (hole \#4, 60s). This pattern of results indicates that the behavior of the experimental animals was linked to the spatio-temporal associations. 
A. Control

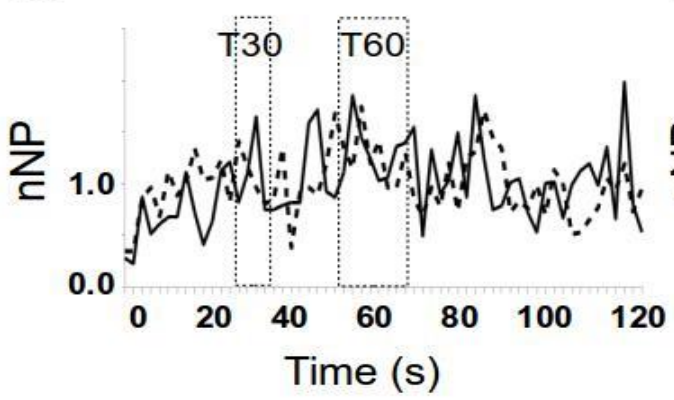

C.

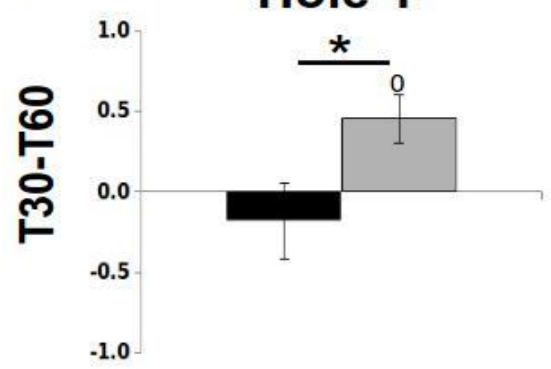

B. Experimental

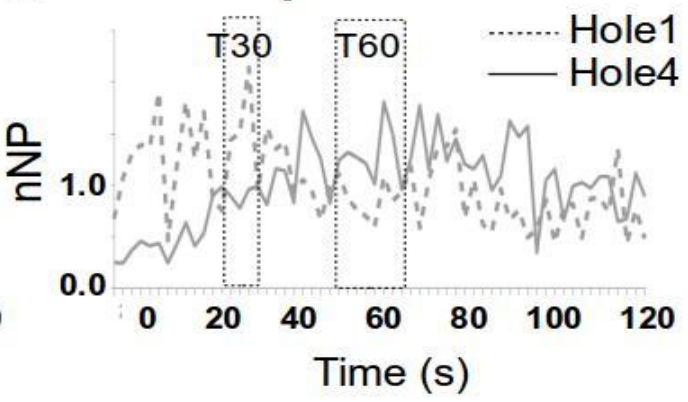

D.

Hole 4

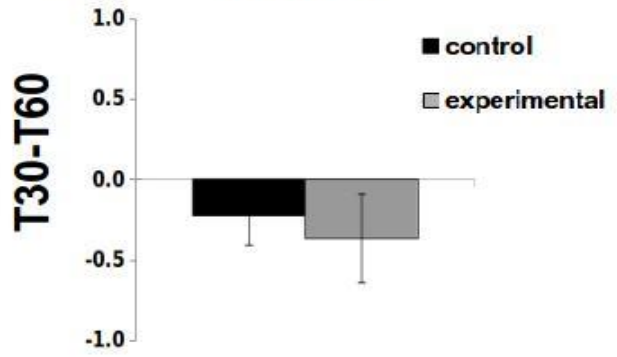

Figure 4: Spatio-temporal binding

The temporal behavior in the new hole (\#1) (dashed line) and old hole (\#4) (solid line) is compared for the control (A) and experimental (B) groups (normalized NP by the overall activity in function of time). For each animal, the mean activity during a temporal window centered at 60 s (old time) was subtracted to the mean activity during a temporal window centered at 30s (new time) for comparison between the two groups for the new (C) and old (D) holes. Significant differences are displayed as: from zero ="“0”and between groups $=$ "**” when $\mathrm{p}<.05$.

In order to assess more specifically the learning of the spatio-temporal association, we compared the mean level of activity in those two holes when restraining to temporal windows centered at the new and old timing rules. For each animal and each hole, the mean activity for the 60-s window (from 52-68s) was subtracted to the mean activity for the 30-s window (from 26-34s) and compared between the two groups for the new and old holes (figures 4C and D). Following this calculation, a behavior linked to a spatio-temporal learning would thus be positive (more activity in the new hole/new time and no differential or less activity in the other combinations new hole/old time, old hole/new time or old hole/old time). For the hole \#1 (new hole) this difference was positive only for the experimental group, indicating that the animals visited more the hole \#1 at 30s (in concordance with the new rule) than at 60s (t-test to zero: $t(5)=3.03, p=.029$ ). However, no such 
difference was observed for the control group $(t(5)=-0.75, p=0.49)$ confirming that the animals did not have a discernible temporal behavior in this hole. This analysis also revealed a difference between the two groups $(t(10)=2.249, p=.048)$ in this hole (figure $4 \mathrm{C})$. The same analysis for the old hole (hole \#4, figure 4D) showed no differential spatio-temporal behavior (difference from zero, $(t(5)=-1.33, p=.24$ and $t(5)=-1.33, p=.24$ for control and experimental groups), and no statistical differences between the two groups $(t(10)=0.70, p=.50)$.

\section{Day +1 after the Shift of the spatio-temporal association - Context A}

The context A was not associated to a change of the spatio-temporal rule. Therefore, no change was expected in the behavior of the animals, and no difference between the two groups either. Indeed, as shown in the figure 5, all the animals kept an adjusted spatio-temporal behavior. With regard to their spatial behavior, the animals visited mainly the hole \#2, reinforced in this context, but showed also a slight increased activity in the hole newly reinforced in the other context $\mathrm{B}$, hole \#1 (figure 5A). We focused our analyses on these two holes (\#2 and \#1). There was no significant difference between the two groups in neither of these two holes $(t(10)<1.31$, ns $)$. Despite the fact that the spatial rule had not been changed in this context, the animals decreased significantly their visits in the hole \#2 $(t(10)=-5.45, p<.001)$, and increased their visits in the hole $\# 1(t(10)=9.25, p<.001)$, compared to their behavior before phase 2 . The numbers of visits in the holes \#3, \#4 and \#5 were not significantly different from before the phase 2 (respectively: $t(10)=$ $2.03, t(10)=2.17, t(10)=.08 ; p s>.05)$.

However, with regard to the temporal behavior in holes \#1 and \#2 (depicted in figure 5BC), all the animals showed an increased activity centered at the peak interval time for this context (15s) in both holes (significant effect of time, $F(44,440)=1.75, p<.01$ for hole \#1, and $F(44,440)=8.31$, $p<.001$ for hole \#2; and no hole $\mathrm{x}$ time interaction in either case $F s<1.14$, ns,), with no difference 
312 between groups (no group $\mathrm{x}$ time interaction, $F s<1$, ns confirmed by a Bayesian analysis, with the 313 main effect model preferred to the interaction model by a Bayesian factor of 148.77 for hole \#1 and 31475.83 for hole \#2).
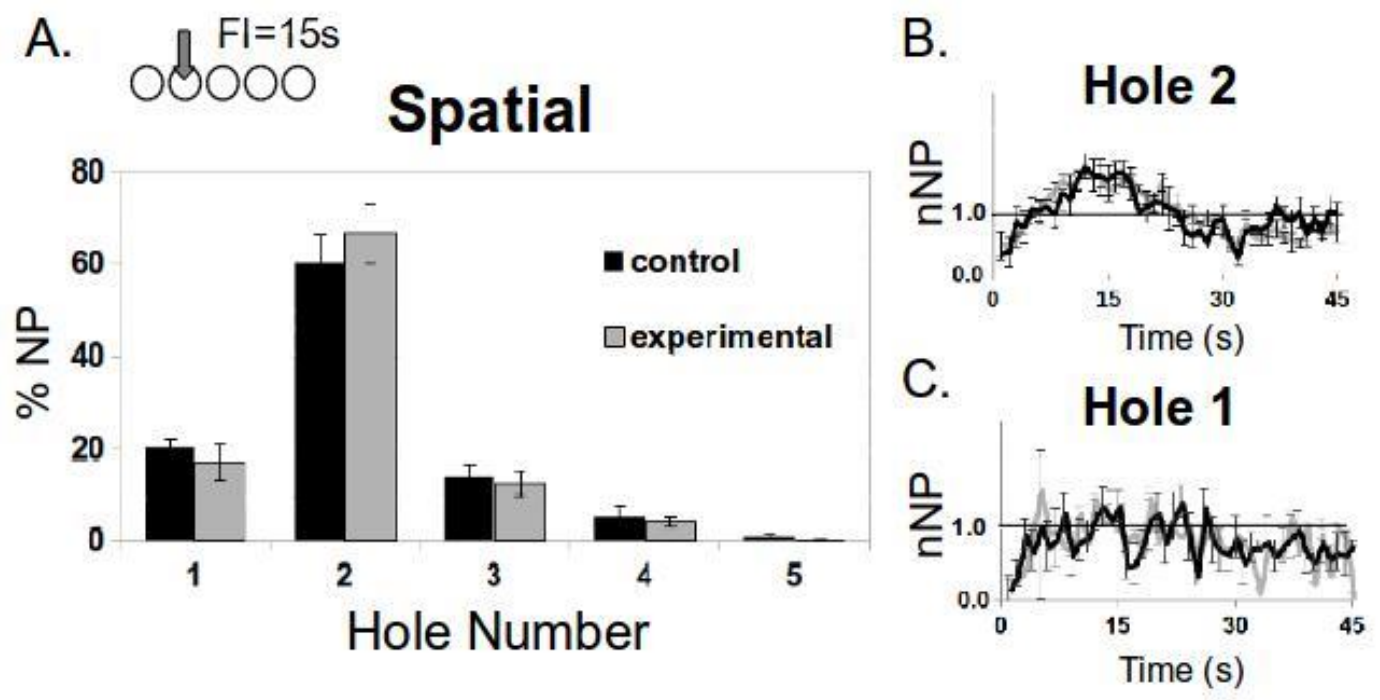

Figure 5: Impact of the shift session at Day+1 in context A

(A) Spatial activity ( $\% \mathrm{NP}+/-\mathrm{SE})$ at day 1 after phase 2 for all the holes in context A. Temporal behavior for the two groups in the hole of interest \#2 corresponding hole. 


\section{Context B}

A.

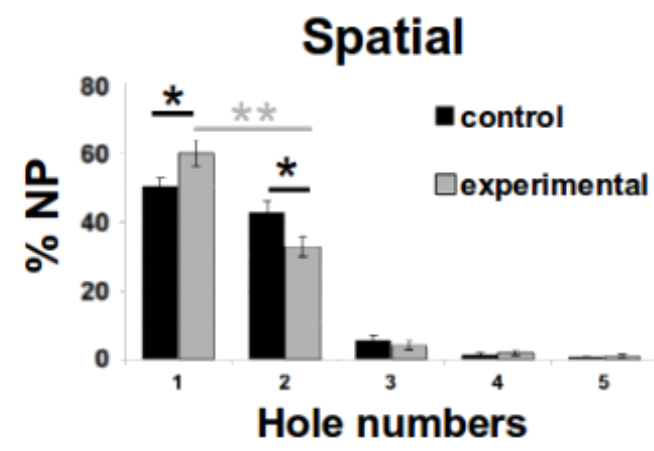

Context A

D.

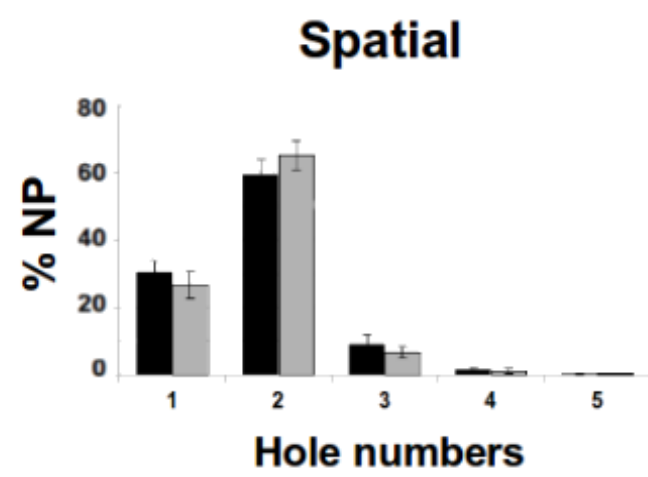

B. Hole1

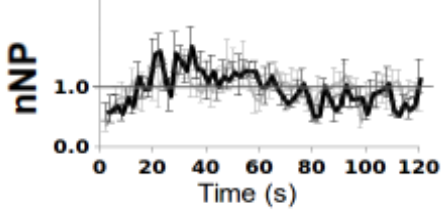

C. Hole2

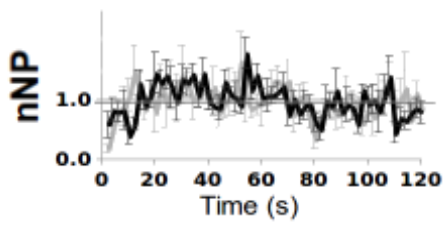

Figure 6: Impact of the shift session at Day+9 in the two contexts

(A) Percentages of Nose-Pokes $(\% \mathrm{NP}+/-\mathrm{SE})$ for each of the holes in context $\mathrm{B}$, for the control (black) and the experimental (grey) groups. Between groups (black, ${ }^{*} \mathrm{p}<.05$ ) and within group (grey, $\left.{ }^{* *} \mathrm{p}<.001\right)$ analyses focusing on holes \#1 and \#2, reinforced holes in context B and A, respectively. Normalized temporal activity in context B (nNP +/-SE) in function of time) in holes \#1 (B) and \#2 (C) for control (black) and experimental (grey) groups. (D) Percentages of Nose-Pokes $(\% \mathrm{NP}+/-\mathrm{SE})$ for each of the holes in context A, for the control (black) and the experimental (grey) groups. Temporal activity (NP +/-SE) in function of time in context A in holes \#1 (E) and \#2 (F) for control (black) and experimental (grey) groups.

First, in both contexts (figure 6A \& D), there was still a spatial generalization to the hole reinforced in the other context (around 20-40\%), whereas the activity in hole \#4 was extinguished. Second, interestingly, the benefit of the shift session was visible on the spatial behavior in context B

335 (figure 6A), as the experimental group showed a higher level of activity in hole \#1 compared to hole $\# 2(t(5)=-4.48, p=.007)$, whereas it was not the case for the control group $(t(5)=-1.33, p=.24)$. This differential behavior was confirmed with between group differences with higher activity for the 
experimental group in the reinforced hole \#1 $(t(10)=2.20, p=.026)$ and lower activity for the unreinforced hole $\# 2$ in this context $(t(10)=2.19, p=.027)$, as compared to the control group. No differential spatial behavior was observed in context A (figure 6D), with a similar level of activity between groups for both hole \#1 and \#2 $(t(10)<1.50$, ns). These results indicate that the control animals did not differentiate the hole \#1 from the hole \#2 in context B, showing that they did not yet fully learn the new spatial component of the rule, contrarily to the experimental group who showed an adjusted spatial behavior in this task.

The temporal behavior did not differ between groups in neither hole \#1 or \#2 for both contexts (no significant group x time interaction, $F_{\mathrm{s}}(59,590)<1$, ns with a Bayes factor of 33.37 for $\# 1$ and of 23.54 for \#2 in favor of a main effect for context B ; Fs $(44,440) \leq 1.0$, ns for context A, also confirmed by a Bayes factor of 4.58 for hole \#1 and 242.86 for hole \#2). Both groups showed an activity peaking around $30 \mathrm{~s}$ in context $\mathrm{B}$, and around $15 \mathrm{~s}$ in context $\mathrm{A}$, with a sharper temporal function in the reinforced hole (hole\#1 in context $\mathrm{B}$, and hole \#2 in context $\mathrm{A}$ ) than in the nonreinforced holes. These results show a correct learning of the temporal rule for both groups that is well controlled by the context.

\section{Discussion}

The aim of the present study was to investigate the learning of space and time when both dimensions are associated and to assess whether the two dimensions are learnt independently of each other. For this purpose, we developed an experimental paradigm based on a nose-poke setup and peak-interval procedure to assess the learning of a new spatio-temporal association, once the rules have been acquired. The main results showed (1) a spatio-temporal binding, which is conserved when the rule is changed, and a fast and robust adaptation to the new associative rule; (2) 
361 a faster learning of the timing rule which is not dissociable neither from a spatial nor a contextual 362 dimension.

After learning the two spatio-temporal associations in each context during Phase 1, the animals were subjected once to a shift of this rule (Phase 2). They benefited from this single session (or shift of the spatio-temporal rule) in adapting faster to this new rule in Phase 3 . Indeed, we observed that the experimental animals expressed a differential spatio-temporal binding according to both the old rule (hole \#4,60s) and the new rule (hole \#1, 30s), indicating that the spatial and temporal components of the rule had been learned bound together, in a coherent association within the context. At the same time, the spatio-temporal association learned in context A (hole \#2 reinforced at 15s) was spared, which confirms that the animals were able to discriminate between the two contexts, despite a slight spatial generalization to the hole \#1. training, as only the experimental group presented the appropriate spatio-temporal behavior (hole $\# 1,30$ s, in context B). The spatial generalization observed for the control group suggests that the spatial component might be learned later in comparison to the timing rule, as we will discuss below.

\section{Spatio-temporal binding and adaptation to the change}

In this experiment, we submitted the animals to a shift of the spatio-temporal rule after the of location and time, independently of the acquisition of the instrumental task. The main question was then: would one dimension shift before the other, or would the two dimensions be learned at the same rate? 
The results showed a facilitated adaptation to a change in an associative rule combining place

and interval time learning. The pattern of results indicates that a single session was sufficient for the animal to learn a new spatio-temporal association. First and foremost, this result supports the work of our colleagues (Molet and Miller, 2013; Buhusi, 2014; Tosun et al, 2016) by confirming that animals are able to encode the location and temporal attributes of a given situation. Secondly, this study shows that interval time can be learnt fast which echoes the findings of several studies showing that animals can learn time in one session. For example, Guilhardhi and Church (2005) showed that rats were able to adapt quickly to repeated shifts in a PI task. Similarly, Dallérac et al (2017) observed that rats can exhibit fast adaptation of temporal fear conditioned responses when changing the time between the CS and US in a conditioned suppression task. Other reports have shown that time is learned in a single trial (Diaz-Mataix et al, 2013; Davis et al, 1989).Recent research also reported that mice are able to adjust their temporal behavior quickly and abruptly (only one trial) to a change of reinforcement rule, by using probabilistic estimate of the rule (Kheifets and Gallistel 2012). In a comparable manner, it is well known that animals can rapidly adapt to changes in spatial representation. For example, animals are able to rapidly adapt to changes in spatial memory tasks, accompanied with a remapping of the hippocampal place cells (O'Keefe and Dostrovsky, 1976; Morris, 1986; Moser et al, 2008; for review see Eichenbaum et al, 1999; Hartley et al , 2013). In addition, a recent study in monkeys by Kowk and collaborators (2015) confirmed that animals can exhibit flexibility to rapidly adapt to spatio-temporal changes in a delayed matching-to-position task. Our results thus comfort the previous results of the literature, extending it further. In sum, our present findings confirm that rodents do have the capacity to perceive and adjust quickly their behavior to a complex change of a rule, supporting the view that animal may represent time and space in their memory, therefore offering a framework for decision making, as suggested in a recent review (Gur et al, 2108). 
More importantly, our results show that when learned in association, there is a spatio-

temporal binding formed that governs behavior in a selective manner according to each of the old and new rules. Surprisingly, though, the behavior of the control animals was not spatially adjusted 8 days after the shift, while their temporal behavior was not significantly different from the experimental animals in the new reinforced location (hole \#1). The imprecision in nose-poke choice might be due to the fact that, in phase 3, all nose-pokes were lit. In a similar situation, Tosun et al (2016) showed that when two previously reinforced locations are lit at the same time, the animals explore the two locations with a switching behavior between the two locations governed by the temporal rules associated with them. Furthermore, the behavior of experimental animals in the present experiment clearly shows that time and space were bound together (see figure 4). The fact that the temporal pattern of the visits to the hole \#2 was not related to its specific association in the other context, nor to the old or new temporal rule in this context (see figure 6), suggests that the animal's behavior reflected some imprecision in spatial memory (as also suggested by the activity in hole \#3). Nevertheless, the results may indicate that the spatial dimension needs more time to be learned than the temporal one. One may argue, however, that the situation was not symmetrical for

422 both dimensions, as the long-to-short shift in the temporal dimension could be seen as without ambiguity for the animal (because reinforcement is available earlier than expected) contrary to the

424 spatial situation where all possible locations were lit. Further experiments will be needed to 425 question the hierarchical organization in memory of spatial and temporal representations. In the 426 framework of working memory, an asymmetry of the representation of spatial and temporal 427 information has been found regarding the type of task and/or the sensory modality engaged (Buhusi 428 et al, 2013; Noyce et al, 2015; Michalka et al, 2015). Gallistel (2011) proposed that abstract 429 dimensions such as time, space and numbers could be represented as magnitudes. But little is 430 known about the link of this representation within a contextual event and the formation of a meaningful memory articulating those parameters together. 
results that when the association is presented to the animals in an explicit manner (with a Pavlovian

shift of the rule), time and space are learned together, exhibiting spatio-temporal binding. However, without precise indications, such as visual cues, time may be learnt before space.

\section{Binding of time and space within context}

In the present work, in addition to the spatio-temporal association, the animals had to learn

the difference between two different contexts (A and B) which differed by the lighting of the experimental box and the frequency of the discriminative timing stimuli (tone). While this situation

provides a control for the specificity of the obtained effects, it also gives some indication of whether

there had been some tripartite (time-location-context) memory formed. The rats' ability to learn a

complex association of space-event-context has been well documented in many behavioral

paradigms assessing episodic-like memory in rodents, and is referred to as the what/where/which context association (for similar studies, see Eacott and Norman, 2004; Eacott et al, 2005; Easton and Eacott 2008; Eacott and Easton 2010). A recent work from Crystal and Smith (2014) also reported that rats can dissociate episodic events based on contextual cues in order to form a bound representation of multiple dimensions of the memory. But these studies focused on episodic-like memory, and used a very different time scale than the second-to-minute range. To our knowledge, no experiment has studied a contextual dimension in the framework of interval timing. the animals were able to discriminate between the two contexts during the initial training (Phase 1). In addition, the representation of the context appeared to be conserved in Phase 3, as shown by an 454 appropriate spatio-temporal behavior in context A (hole \#2-15s) and the correct adaptation to the 455 new rule of the context $\mathrm{B}$ (hole \#1-30s) by the experimental animals. Nevertheless, two 
456 observations can be made. First, while the spatio-temporal rule was not changed in context A, a 457 slight increase in visits was observed in the hole \#1 in this context (hole newly reinforced in context 458 B). Second, the hole \#2 (reinforced in context A) was still visited in context B even at the end of 459 phase 3 (especially in control animals), although it had never been reinforced in that context. Thus, 460 there was some generalization of the spatial learning between the two contexts despite their very 461 different characteristics, especially the highly discriminable timing cues (1 kHz vs. $11 \mathrm{kHz})$. One 462 may suspect that the fact that all the nose-poke holes were lit in Phase 3 may have played a role, 463 although proximity between the holes could not be the sole factor, as no such increase in responding 464 was found in the opposite side, equally distant to hole \#2 (i.e., hole \# 3). Importantly, this 465 uncertainty was weakened by the Pavlovian shift session in the experimental animals. Furthermore, 466 the temporal behavior was properly adjusted to the context from day one in the experimental 467 animals, suggesting that a time-context association is learned faster than a space-context 468 association. Further experiments will be necessary to clearly compare the speed of learning of each 469 of the components of the space-time-context triplet, and the rules governing their binding.

471 Neuronal representation of the association

473 time represented in the same anatomical structures? Up to date, two brain areas have been reported 474 to support spatial and interval timing processes.

476 timing and temporal processes (for a review see Buhusi and Meck, 2005). A rapid adaptation to a 477 change of rule has been previously linked to a change in the firing rate of the task-modulated striatal 478 neurons (Portugal et al, 2011). The author of this study suggested that rather than event times, the 479 striatum might encode the timing of adaptive behavioral transitions between options based on the 
environmental statistics. Similarly, Mello et al (2015) demonstrated in an electrophysiological experiment that striatal neuronal populations can quickly adapt to the immediate task demands by rescaling their temporal tuning to abrupt changes in delays to reinforcement, similar to our present task. Furthermore, the striatum has been reported to be involved in spatial memory; for example, it has been shown that lesions in the dorsal part of the striatum leads to deficits in spatial learning (Block et al, 1993; McDonald and White, 1994; Lee et al, 2014). A recent study in rats also confirmed that a unilateral lesion of this structure can lead to a disruption of spatio-temporal integration (Blankenship et al, 2017), which may lead to think that the dorsal striatum might be involved in our present task.

Secondly, the hippocampal formation has been extensively studied regarding spatial navigation (O'Keefe and Dostrovsky, 1971; Save et al, 1992; Maguire et al, 1997; GoodrichHunsaker et al, 2008). In addition, it is known that the hippocampal place cells are able to code for interval time as well (Pastalkova et al, 2008; MacDonald et al, 2011; Kraus et al, 2013) and that fornix lesions (initially found to impair spatial learning) impair the detection of temporal changes (Kwok et al, 2015). The involvement of hippocampus in interval timing has been highlighted in a recent study (Jacobs et al, 2013), although in a supra-minute time range. Another recent finding also suggests that hippocampus and memory-related mechanisms are involved in spatio-temporal integration bringing evidence that the hippocampi are critical components of internal clock mechanism (Buhusi et al (2013). All these results are clues pointing into the direction that the hippocampal formation might also be involved in the spatio-temporal binding that we report in our present study, and more research needs to be conducted to further clarify this question.

In conclusion, using a behavioral paradigm that enables the study of the interaction of space, interval time and context, and their overall binding, we have found differential dynamics of space 
and interval time learning, and a tripartite space-time-context binding. The matters of probabilistic estimate of rules, animal cognitive representation and behavioral adaptation to a change of rule still demand more investigation. We believe that our experimental design can be a basis for further investigations related to the hierarchical learning of such a complex association. We hope that it will foster to a better understanding of the neural mechanisms supporting the integrated representation of space, interval time and context, and therefore contribute to the development of more refined therapeutic approaches for memory impairment and related diseases.

\section{REFERENCES}

Allman, M. J., Teki, S., Griffiths, T. D., \& Meck, W. H. (2014). Properties of the internal clock: first- and secondorder principles of subjective time. Annual Review of Psychology, 65, 743-771. https://doi.org/10.1146/annurevpsych-010213-115117

Aristotle . Book IV Physics ( Delta 208-223b)

Balsam, P. D., Drew, M. R., \& Yang, C. (2002). Timing at the start of associative learning. Learning and Motivation, 33(1), 141-155. https://doi.org/10.1006/lmot.2001.1104

Blanco, E., Santamaría, J., Chamizo, V. D., \& Rodrigo, T. (2006). Area and peak shift effects in a navigation task with rats. International Journal of Psychology and Psychological Therapy, 6(3).

Blankenship, P.A., Cheatwood, J.L., \& Wallace, D.G. (2017). Unilateral lesions of the dorsocentral striatum (DCS) disrupt spatial and temporal characteristics of food protection behavior. Brain Struct Funct. 222(6):26972710. doi: 10.1007/s00429-017-1366-6.

Block,F., Kunkel, M., \& Schwarza, M. (1993). Quinolinic acid lesion of the striatum induces impairment in spatial learning and motor performance in rats. Neuroscience Letters, 149(2), 126-128. https://doi.org/10.1016/0304-3940(93)90752-7

Bouton, M. E., \& Bolles, R. C. (1979). Role of conditioned contextual stimuli in reinstatement of extinguished fear. Journal of Experimental Psychology. Animal Behavior Processes, 5(4), 368-378. 
Buhusi, C. V. (2014). Associative and temporal learning: new directions. Behavioural Processes, 101, 1-3. https://doi.org/10.1016/j.beproc.2014.01.005

Buhusi, C. V., \& Meck, W. H. (2005). What makes us tick? Functional and neural mechanisms of interval timing. Nature Reviews. Neuroscience, 6(10), 755-765. https://doi.org/10.1038/nrn1764

Buhusi, C. V., \& Meck, W. H. (2006). Time sharing in rats: A peak-interval procedure with gaps and distracters. Behavioural Processes, 71(2-3), 107-115. https://doi.org/10.1016/j.beproc.2005.11.017

Buhusi, M., Scripa, I., Williams, C. L., \& Buhusi, C. V. (2013). Impaired interval timing and spatial-temporal integration in mice deficient in CHL1, a gene associated with schizophrenia. Timing \& Time Perception, 1, 21-38.

Casasanto, D., \& Boroditsky, L. (2008). Time in the mind: Using space to think about time. Cognition, 106, 579593.

Church, R. M. (2014). A resolution of the debate about associative and temporal learning. Behavioural Processes, 101, 163-165. https://doi.org/10.1016/j.beproc.2013.08.011

Church, R. M., Getty, D. J., \& Lerner, N. D. (1976). Duration discrimination by rats. Journal of Experimental Psychology. Animal Behavior Processes, 2(4), 303-312.

Crystal, J. D. (2009). Theoretical and conceptual issues in time-place discrimination. The European Journal of Neuroscience, 30(9), 1756-1766. https://doi.org/10.1111/j.1460-9568.2009.06968.x

Crystal, J. D., \& Smith, A. E. (2014). Binding of episodic memories in the rat. Current Biology: CB, 24(24), 2957-2961. https://doi.org/10.1016/j.cub.2014.10.074

Dallérac, G., A COMPLETER

Davis, M., Schlesinger, L. S., \& Sorenson, C. A. (1989). Temporal specificity of fear conditioning: effects of different conditioned stimulus-unconditioned stimulus intervals on the fear-potentiated startle effect. Journal of Experimental Psychology. Animal Behavior Processes, 15(4), 295-310.

Devenport, J. A., Luna, L. D., \& Devenport, L. D. (2000a). Placement, Retrieval, and Memory of Caches by Thirteen-Lined Ground Squirrels. Ethology, 106(2), 171-183. https://doi.org/10.1046/j.1439-0310.2000.00522.x Devenport, J. A., Luna, L. D., \& Devenport, L. D. (2000b). Placement, Retrieval, and Memory of Caches by Thirteen-Lined Ground Squirrels. Ethology, 106(2), 171-183. https://doi.org/10.1046/j.1439-0310.2000.00522.x Diana, R. A., Yonelinas, A. P., \& Ranganath, C. (2013). Parahippocampal cortex activation during context reinstatement predicts item recollection. Journal of Experimental Psychology. General, 142(4), 1287-1297. https://doi.org/10.1037/a0034029 
Díaz-Mataix, L., Ruiz Martinez, R. C., Schafe, G. E., LeDoux, J. E., \& Doyère, V. (2013). Detection of a temporal error triggers reconsolidation of amygdala-dependent memories. Current Biology: CB, 23(6), 467-472. https://doi.org/10.1016/j.cub.2013.01.053

Eacott, M. J., \& Easton, A. (2010). Episodic memory in animals: remembering which occasion. Neuropsychologia, 48(8), 2273-2280. https://doi.org/10.1016/j.neuropsychologia.2009.11.002

Eacott, M. J., Easton, A., \& Zinkivskay, A. (2005). Recollection in an episodic-like memory task in the rat. Learning \& Memory (Cold Spring Harbor, N.Y.), 12(3), 221-223. https://doi.org/10.1101/lm.92505

Eacott, M. J., \& Norman, G. (2004). Integrated memory for object, place, and context in rats: a possible model of episodic-like memory? The Journal of Neuroscience: The Official Journal of the Society for Neuroscience, 24(8), 1948-1953. https://doi.org/10.1523/JNEUROSCI.2975-03.2004

Eichenbaum, H., Dudchenko, P., Wood, E., Shapiro, M., \& Tanila, H. (1999). The hippocampus, memory, and place cells: is it spatial memory or a memory space? Neuron, 23(2), 209-226.

Fortin, N. J., Agster, K. L., \& Eichenbaum, H. B. (2002). Critical role of the hippocampus in memory for sequences of events. Nature Neuroscience, 5(5), 458-462. https://doi.org/10.1038/nn834

Gallistel, C. (2011). Space, Time and Number in the Brain: Searching for the Foundations of Mathematical Thought - Mental Magnitudes. Academic Press.

Gould, K. L., Kelly, D. M., \& Kamil, A. C. (2010). What scatter-hoarding animals have taught us about smallscale navigation. Philosophical Transactions of the Royal Society B: Biological Sciences, 365(1542), 901-914. https://doi.org/10.1098/rstb.2009.0214

Gur, E., Duyan, Y., Balc1, F. (2018). Spontaneous Integration of Temporal Information: Implications for Representational/Computational Capacity of Animals. Animal Cognition. 21:3-19

Jacobs, N.S., Allen, T.A., Nguyen, N., Fortin, N.J. (2013). Critical role of the hippocampus in memory for elapsed time. Journal of Neurosci.,33(34):13888-93. doi: 10.1523/JNEUROSCI.1733-13.2013.PMID: 23966708

Kheifets A, Gallistel C (2012) Mice take calculated risks. Proc Natl Acad Sci USA 109(22): 8776-8779.

Kraus completer

Maguire, E. A.;,Frackowiak, R. S. J., \& Frith, C. D. (1997). Recalling routes around London: Activation of the right hippocampus in taxi drivers. The Journal of Neuroscience. 17 (18): 7103-7110. Guilhardi, P., \& Church, R. M. (2005). Dynamics of temporal discrimination. Learning \& Behavior, 33(4), 399-416.

Hartley, T., Lever, C., Burgess, N., \& O'Keefe, J. (2014). Space in the brain: how the hippocampal formation supports spatial cognition. Philosophical Transactions of the Royal Society of London. Series B, Biological Sciences, 369(1635), 20120510. https://doi.org/10.1098/rstb.2012.0510 
Holder, M. D., \& Roberts, S. (1985). Comparison of timing and classical conditioning. Journal of Experimental Psychology. Animal Behavior Processes, 11(2), 172-193.

Jacobs, L. F. (1992). Memory for cache locations in Merriam's kangaroo rats. Animal Behaviour, 43(4), 585-593. https://doi.org/10.1016/S0003-3472(05)81018-8

Jacobs, L. F., \& Liman, E. R. (1991). Grey squirrels remember the locations of buried nuts. Animal Behaviour, 4l(1), 103-110. https://doi.org/10.1016/S0003-3472(05)80506-8

Kesner, R. P., Gilbert, P. E., \& Barua, L. A. (2002). The role of the hippocampus in memory for the temporal order of a sequence of odors. Behavioral Neuroscience, 116(2), 286-290.

Kwok, S. C., Mitchell, A. S., \& Buckley, M. J. (2015). Adaptability to changes in temporal structure is fornixdependent. Learning \& Memory (Cold Spring Harbor, N.Y.), 22(8), 354-359. https://doi.org/10.1101/lm.038851.115

Latimer, K. W., Yates, J. L., Meister, M. L. R., Huk, A. C., \& Pillow, J. W. (2015). NEURONAL MODELING. Single-trial spike trains in parietal cortex reveal discrete steps during decision-making. Science (New York, N.Y.), 349(6244), 184-187. https://doi.org/10.1126/science.aaa4056

Lavenex, P., \& Lavenex, P. B. (2006). Spatial relational memory in 9-month-old macaque monkeys. Learning \& Memory (Cold Spring Harbor, N.Y.), 13(1), 84-96. https://doi.org/10.1101/lm.97606

Lee, A. S., André, J. M., \& Pittender, C. (2014). Lesions of the dorsomedial striatum delay spatial learning and render cue-based navigation inflexible in a water maze task in mice. Front Behav Neurosci. 2014; 8: 42. doi: 10.3389/fnbeh.2014.00042

Lejeune, H., Ferrara, A., Simons, F., \& Wearden, J. H. (1997). Adjusting to changes in the time of reinforcement: peak-interval transitions in rats. Journal of Experimental Psychology. Animal Behavior Processes, 23(2), 211231.

Lejeune, H., Richelle, M., \& Wearden, J. H. (2006). About Skinner and time: behavior-analytic contributions to research on animal timing. Journal of the Experimental Analysis of Behavior, 85(1), 125-142.

MacDonald, C. J., Lepage, K. Q., Eden, U. T., \& Eichenbaum, H. (2011). Hippocampal "time cells" bridge the gap in memory for discontiguous events. Neuron, 71(4), 737-749. https://doi.org/10.1016/j.neuron.2011.07.012

MacDonald, R. J., \& White, N.M. (1994) Parallel information processing in the water maze: Evidence for independent memory systems involving dorsal striatum and hippocampus. Behavioral and Neural Biology, 66 (3), 260-270. https://doi.org/10.1016/S0163-1047(05)80009-3

Maguire, E. A., Frackowiak, R. S. J., Frith, C. D. (1997). Recalling routes around London: Activation of the right hippocampus in taxi drivers. The Journal of Neuroscience. 17 (18): 7103-7110. Matell, M. S., Meck, W. H., \& 
623

624

625

626

627

628

629

630

631

632

633

634

635

636

637

638

639

640

641

642

643

644

645

646

647

648

649

650

651

652

653

Nicolelis, M. A. L. (2003). Interval timing and the encoding of signal duration by ensembles of cortical and striatal neurons. Behavioral Neuroscience, 117(4), 760-773.

Meck, W. H., Church, R. M., \& Matell, M. S. (2013). Hippocampus, time, and memory--a retrospective analysis. Behavioral Neuroscience, 127(5), 642-654. https://doi.org/10.1037/a0034201

Meck, W. H., Church, R. M., \& Olton, D. S. (1984). Hippocampus, time, and memory. Behavioral Neuroscience, 98(1), 3-22.

Mello, G. B. M., Soares, S., \& Paton, J. J. (2015). A scalable population code for time in the striatum. Current Biology: CB, 25(9), 1113-1122. https://doi.org/10.1016/j.cub.2015.02.036

Mendl, Laughlin,, \& Hitchcock, . (1997). Pigs in space: spatial memory and its susceptibility to interference. Animal Behaviour, 54(6), 1491-1508. https://doi.org/10.1006/anbe.1997.0564

Michalka, S. W., Kong, L., Rosen, M. L., Shinn-Cunningham, B. G., \& Somers, D. C. (2015). Short-Term Memory for Space and Time Flexibly Recruit Complementary Sensory-Biased Frontal Lobe Attention Networks. Neuron, 87(4), 882-892. https://doi.org/10.1016/j.neuron.2015.07.028

Molet, M., \& Miller, R. R. (2014). Timing: an attribute of associative learning. Behavioural Processes, 101, 4-14. https://doi.org/10.1016/j.beproc.2013.05.015

Morris, R. G. M., Hagan, J. J., \& Rawlins, J. N. P. (1986). Allocentric spatial learning by hippocampectomised rats: A further test of the "spatial mapping" and "working memory" theories of hippocampal function. The Quarterly Journal of Experimental Psychology Section B, 38(4), 365-395.

https://doi.org/10.1080/14640748608402242

Moser, E. I., Kropff, E., \& Moser, M.-B. (2008). Place Cells, Grid Cells, and the Brain's Spatial Representation System. Annual Review of Neuroscience, 31(1), 69-89. https://doi.org/10.1146/annurev.neuro.31.061307.090723

Myers, K. M., \& Davis, M. (2007). Mechanisms of fear extinction. Molecular Psychiatry, 12(2), 120-150. https://doi.org/10.1038/sj.mp.4001939

Noyce, A., Cestero, N., Shinn-Cunningham, B., \& Somers, D. (2015). Space Depends On Time: Informational Asymmetries in Visual and Auditory Short-Term Memory. Journal of vision, 15(12), 1054-1054

O’Keefe, J., \& Dostrovsky, J. (1971). The hippocampus as a spatial map. Preliminary evidence from unit activity in the freely-moving rat. Brain Research, 34(1), 171-175.

Olton, D. S., Collison, C., \& Werz, M. A. (1977). Spatial memory and radial arm maze performance of rats. Learning and Motivation, 8(3), 289-314. https://doi.org/10.1016/0023-9690(77)90054-6

Pastalkova comlterPavlov, I. P. (1927). Conditioned reflexes: London: Oxford UP. 
Piaget, J. (1927/1969). The child's experience of time. New York : Ballantine Books.

675

676

Portugal, G. S., Wilson, A. G., \& Matell, M. S. (2011). Behavioral Sensitivity of Temporally Modulated Striatal Neurons. Frontiers in Integrative Neuroscience, 5, 30. http://doi.org/10.3389/fnint.2011.00030

Rescorla, R. A., \& Heth, C. D. (1975). Reinstatement of fear to an extinguished conditioned stimulus. Journal of Experimental Psychology. Animal Behavior Processes, 1(1), 88-96.

Robin, J., Wynn, J., \& Moscovitch, M. (2016). The spatial scaffold: The effects of spatial context on memory for events. Journal of Experimental Psychology. Learning, Memory, and Cognition, 42(2), 308-315. https://doi.org/10.1037/xlm0000167

Rushworth, M. F. S., \& Behrens, T. E. J. (2008). Choice, uncertainty and value in prefrontal and cingulate cortex. Nature Neuroscience, 11(4), 389-397. https://doi.org/10.1038/nn2066

Sanabria, F., \& Oldenburg, L. (2014). Adaptation of timing behavior to a regular change in criterion. Behavioural Processes, 101, 58-71. https://doi.org/10.1016/j.beproc.2013.07.018

Save, E., Poucet, B., Foreman, N., \& Buhot, M. (1992). Object exploration and reactions to spatial and nonspatial changes in hooded rats following damage to parietal cortex or hippocampal formation. Behavioral Neuroscience. 106 (3): 447-456.. doi:10.1037/0735-7044.106.3.447.

\section{Skinner, B.F., - A Case History in Scientific Method (1956) | Scientific Method | Experiment. (n.d.). Retrieved} August 5, 2017, from https://fr.scribd.com/document/206630017/B-F-Skinner-A-Case-History-in-ScientificMethod-1956

Thorpe, C. M., \& Wilkie, D. M. (2002). Unequal interval time-place learning. Behavioural Processes, 58(3), 157166.

Tosun, T., Gür, E., \& Balc1, F. (2016). Mice plan decision strategies based on previously learned time intervals, locations, and probabilities. Proceedings of the National Academy of Sciences of the United States of America, 113(3), 787-792. https://doi.org/10.1073/pnas.1518316113

Wilkie, D. M., Carr, J. A. R., Galloway, J., Jo Parker, K., \& Yamamoto, A. (1997). Conditional time-place learning. Behavioural Processes, 40(2), 165-170. https://doi.org/10.1016/S0376-6357(97)00781-X 